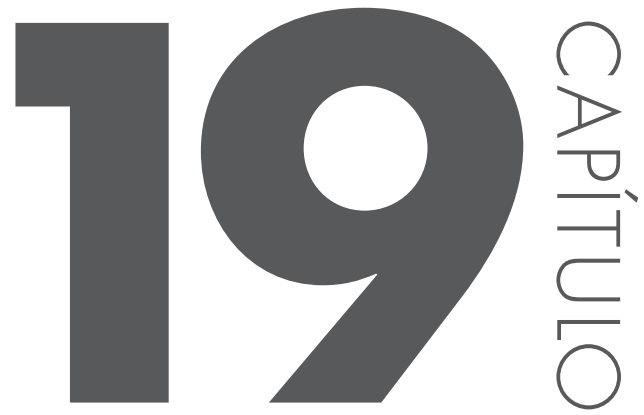

\title{
MÉTODO DE OPERADORES EM MECÂNICA QUÂNTICA
}

Elias Vieira, Lucas Henrique ${ }^{1}$; Ribeiro dos Anjos, Petrus ${ }^{1}$

IInstituto de Física e Química. Universidade Federal de Goiás - Regional Catalão

*email: lucasvieira_15@hotmail.com 
Resumo: O presente artigo irá introduzir e discutir o Método de Operadores em Mecânica Quântica. Essa apresentação será feita tomando-se cuidado com o rigor matemático, mas também motivando fisicamente as definições e proposições estabelecidas.

\section{Palavras-chave: Física; Mecânica Quântica; Operadores.}




\section{1 . Introduc̣ão}

Neste trabalho apresentaremos uma introdução a alguns conceitos matemáticos da mecânica quântica do ponto de vista da teoria dos operadores. Essa apresentaçãa será feita tomando-se cuidado com o rigor matemático, mas também motivando fisicamente as definições e proposições estabelecidas. Nosso trabalho é baseado no estudo sistemático da referência [1].

Este texto está organizado da seguinte forma. Na seção 2, trataremos das bases matemáticas para a descrição quântica de partículas em uma dimensão, trataremos a questão da posição da partícula na subseção 2.1 e discutiremos brevemente a visão probabilística associada a determinação da posição desta partícula. Na subseção 2.2 
será apresentada a ideia de valor esperado. Na subseção 2.3, trataremos do momento linear do ponto de vista quântico. Na subseção 2.4, trataremos da energia relacionada a uma partícula. Na subseção 2.5 introduziremos o conceito de observáveis e analisaremos quais funções são aceitas para função de estado, em qual espaço estas se encontram, e definiremos o que é um espaço fechado ou denso. Apresentaremos nossas conclusões na seção 3 .

\section{Movimento unidimensional}

Nesta seção discutiremos posição, momento e energia de uma partícula do ponto de vista quântico.

\subsection{Posição}

Na Mecânica Clássica estudamos a posição dos corpos, porém, em ordens de grandeza bem pequenas, por exemplo, em uma escala atômica ou subatômica, deixamos esta visão clássica e partimos para uma interpretação probabilística baseada nas chamadas funções de onda. Um objeto nesta escala tem um comportamento dual que contraria a intuição, que chamamos de partícula-onda. Considere, por exemplo, o caso da luz, em sua explicação do efeito fotoelétrico Einstein propôs que a luz é composta de corpúsculos chamados fótons e que a energia relacionada a um fóton de luz de frequência angular $\omega$ é igual a:

$$
E=\hbar \omega,
$$

onde $\hbar=h / 2 \pi$ e $h$ é a constante de Planck e tem valor igual a $h=6,6262 \cdot 10^{-34}(J \cdot s)$

Porém no experimento da dupla fenda de Thomas Young, e.g.[3], ficou bem evidente o comportamento ondulatório da luz. Assim, é necessário, formular uma interpretação que seja capaz de unificar estes dois comportamentos.

O primeiro postulado que será estabelecido é o seguinte: 
Postulado 1- A probabilidade de se encontrar uma partícula em um intervalo $I \mathrm{C} \mathbb{R}$ num instante $\mathrm{t}$, onde $t \in \mathbb{R}$, é dado pela seguinte expressão

$$
\text { Probabilidade em } I=\int_{I}|\Psi(x, t)|^{2} d x \text {, }
$$

onde $\Psi(x, t)$ é uma função de estado associada à partícula.

Este postulado segue da interpretação proposta por Born que interpreta o módulo ao quadrado da função de onda $(\Psi)$ como uma densidade de probabilidade, sendo então a probabilidade dada pela equação $d P(x)=|\Psi(x, t)|^{2} d x$, onde $d P(x)$ é o diferencial de probabilidade de encontrar a partícula em um intervalo infinitesimal $d x$ da reta.

Fenômenos ondulatórios observados por partículas quânticas, como a interferência no experimento da dupla fenda, aparecem devido ao comportamento da função de onda $\Psi$.

Note que $\circ$ postulado 1 não determina a posição da partícula, mas sim a probabilidade de encontrá-la em um intervalo. A probabilidade $P$ de um evento ocorrer pode ser $0 \leq P \leq 1$. É claro que ao procurar uma partícula ao longo de toda a reta, teremos cem por cento de chance de encontrá-la. Matematicamente essa ideia se traduz em:

$$
\int_{-\infty}^{+\infty}|\Psi(x, t)|^{2} d x=1
$$

para cada instante t.

\subsection{Valor Esperado}

Com uma interpretação probabilística, precisamos de ferramentas para determinar o resultado esperado de uma medida. Começaremos a desenvolver estas ideias nesta subseção e as aplicaremos especificamente ao caso da posição de uma partícula. 
Seja $w$ uma quantidade mensurável, que pode assumir os valores $w_{1}, w_{2}, \ldots, w_{N}$. Suponha que o fato de medir um destes valores não interfira na medida do outro, ou seja, que estes são eventos independentes. Vamos denotar por $P_{k}, 1 \leq k \leq N$, a probabilidade de $w$ assumir o valor $w_{k}$. A probabilidade de ocorrer qualquer um entre $\mathrm{N}$ eventos é a soma das $\mathrm{N}$ probabilidades individuais. Logo, $P_{1}+P_{2}+\cdots+P_{N}=1$, pois é certo que teremos um dos valores.

O valor médio de $w$ (também chamado de valor esperado) [4] é dado por

$$
\bar{w}=w_{1} P_{1}+\cdots+w_{N} P_{N} .
$$

Este é o valor esperado ao realizarmos a medida. Note que $\bar{w}$ não precisa ser igual a nenhum dos valores $w_{1}, w_{2}, \ldots, w_{N}$, que são os valores que $w$ pode assumir, 0 resultado é a média ponderada dos valores que $w$ pode assumir. A razão para esta terminologia está associada à lei dos grandes números e expressa no seguinte teorema (para a demonstração ver [4]).

Teorema 2.1 - Suponha a sequência de idênticos experimentos feitos e os valores $S_{1}, S_{2}, \ldots, S_{n}$ são observados (os números $S_{n}$ estão entre os valores $w_{1}, w_{2}, \ldots, w_{N}$ ). Então a média dos valores $\left(S_{1}+S_{2}+\cdots+S_{n}\right) / n$, converge para $\bar{w}$, "no sentido probabilístico".

Em outras palavras, o teorema expressa que para um $\varepsilon>0$, a probabilidade de

$$
\left|\frac{1}{n}\left(S_{1}+S_{2}+\cdots+S_{n}\right)-\bar{w}\right|>\varepsilon
$$

tende a zero se $n \rightarrow \infty$. É claro, este não é o sentido usual do termo convergência. No "sentido probabilístico", que estamos discutindo, dizemos que a chance da média das medidas ser muito diferente de $\bar{w}$ é pequena. 
Considere agora $o$ caso onde $w$ pode assumir qualquer valor em um intervalo $I$. intervalo $I$ pode ser dividido em subintervalos $I_{k}$ e podemos escolher $x_{k} \in I_{k}$ um ponto arbitrário destes subintervalos. Denotemos $P_{I k}$ é a probabilidade da medida se encontrar no subintervalos $I_{k}$, e consideremos a soma

$$
\sum x_{k} P_{I k}
$$

se esta soma converge no limite em que os subintervalos $I_{k}$ tendem a zero, então nós denominamos o limite desta soma como sendo o valor esperado $\bar{w}$ de $w$.

Para ilustrar a aplicação desta ideia, considere uma partícula descrita pela função de onda $\Psi(x, t)$. Do Postulado $1, P_{I}=\int_{I}|\Psi(x, t)|^{2} d x$ é a probabilidade de encontrar a partícula em um intervalo $I$. Então o valor médio da posição é dado por:

$$
\bar{x}=\lim _{I_{k} \rightarrow 0} \sum x_{k} \int_{I k}|\Psi(x, t)|^{2} d x
$$

onde $I_{k}$ é um subintervalo de $I$, e $x_{k} \in I_{k}$. É claro que este resultado será válido se o limite existir. Isso nos leva ao seguinte resultado:

Lema 2.1 - Se $\Psi(x, t)$ é contínua para $x$ em $I$, então (6) converge para

$$
\int_{I} x|\Psi(x, t)|^{2} d x
$$

com o máximo comprimento de intervalo $I_{k}$ tendendo a zero independentemente da escolha de $x_{k}$ e $I_{k}$.

Demonstração do Lema 2.1: Podemos escrever

$$
\left.\left|\int_{I} x\right| \Psi(x, t)\right|^{2} d x-\sum x_{k} \int_{I_{k}}|\Psi(x, t)|^{2} d x|=| \sum \int_{I_{k}}\left(x|\Psi(x, t)|^{2}-x_{k}|\Psi(x, t)|\right) d x \mid .
$$


Usando a desigualdade triangular [5] temos que:

$$
\left.\left|\int_{I} x\right| \Psi(x, t)\right|^{2} d x-\sum x_{k} \int_{I_{k}}|\Psi(x, t)|^{2} d x\left|\leq \sum \int_{I k}\right| x-\left.x_{k}|| \Psi(x, t)\right|^{2} d x
$$

Dado $\varepsilon>0$, tomemos o comprimento de $I_{k}<\varepsilon$, portanto se $x \in I_{k}$ temos $\left|x-x_{k}\right|<\varepsilon$. Aproximando $x$ de $x_{k^{\prime}}$ teremos que o lado direito se torna tão pequeno tanto quanto se queira, oque mostra a convergência do termo da esquerda da equação (7). Em consequência do Lema 2.1 podemos enunciar imediatamente o:

Teorema 2.2 - Se $\Psi(x, t)$ é uma função contínua em $x$ para cada instante $t$ e $\int_{-\infty}^{\infty}|x||\Psi(x, t)|^{2} d x<\infty$, então o valor médio da posição é dada por

$$
\bar{x}=\int_{-\infty}^{\infty} x|\Psi(x, t)|^{2} d x
$$

Demonstração do Teorema 2.2 - Suponha que as posições de n partículas idênticas sejam medidas em um tempo $t$, e que todas sejam descritas pela mesma função de onda. Sejam $x_{1}, x_{2}, \ldots, x_{n}$ os valores medidoss da posição. Dado um $x_{0} \in \mathbb{R}$ e $a>0$, a probabilidade de que $\left|x_{o}-\sum_{k=1}^{n} x_{k}\right|>a$ é dada por

$$
\int_{M}\left|\Psi\left(x_{1}, t\right) \ldots \Psi\left(x_{n}, t\right)\right|^{2} d x_{1} \ldots d x_{n}
$$

onde $M=\left|x_{o}-\sum x_{k}\right|>a$. Em $M, a^{-2}\left|x_{o}-\sum x_{k}\right|^{2}>1$, e portanto a Eq.(9) é limitada superiormente por 
$a^{-2} \int_{-\infty}^{\infty}\left|x_{o}-\sum x_{k}\right|^{2}\left|\Psi\left(x_{1}, t\right) \ldots \Psi\left(x_{n}, t\right)\right|^{2} d x_{1} \ldots d x_{n}$

desenvolvendo a Eq. (10) teremos

$$
\begin{gathered}
a^{-2} \int_{-\infty}^{\infty}\left(x_{0}^{2}-2 x_{0} \sum x_{k}+\sum x_{k} x_{j}\right)\left|\Psi\left(x_{1}, t\right) \ldots \Psi\left(x_{n}, t\right)\right|^{2} d x_{1} \ldots d x_{n} \\
=a^{-2} \int_{-\infty}^{\infty} x_{0}^{2}\left|\Psi\left(x_{1}, t\right) \ldots \Psi\left(x_{n}, t\right)\right|^{2} d x_{1} \ldots d x_{n} \\
+a^{-2} \int_{-\infty}^{\infty}\left(-2 x_{0} \sum x_{k}\right)\left|\Psi\left(x_{1}, t\right) \ldots \Psi\left(x_{n}, t\right)\right|^{2} d x_{1} \ldots d x_{n} \\
+a^{-2} \int_{-\infty}^{\infty}\left(\sum x_{k} x_{j}\right)\left|\Psi\left(x_{1}, t\right) \ldots \Psi\left(x_{n}, t\right)\right|^{2} d x_{1} \ldots d x_{n}
\end{gathered}
$$

o primeiro termo no lado direito da Eq. (11) pode ser reescrito como

$$
a^{-2} x_{o}^{2} \int_{-\infty}^{\infty}\left|\Psi\left(x_{1}, t\right)\right|^{2} d x_{1} \ldots \int_{-\infty}^{\infty}\left|\Psi\left(x_{n}, t\right)\right|^{2} d x_{n}
$$

Da Eq. (3) temos que o que resta desta expressão é $a^{-2} x_{o}^{2}$.

Desenvolvendo o segundo termo da Eq. (1 1) teremos

$a^{-2} \int_{-\infty}^{\infty}\left(-2 x_{o} \sum x_{k}\right)\left|\Psi\left(x_{1}, t\right) \ldots \Psi\left(x_{n}, t\right)\right|^{2} d x_{1} \ldots d x_{n}$

$$
\begin{gathered}
=-2 x_{0} a^{-2} \int_{-\infty}^{\infty}\left(x_{1}+\cdots+x_{n}\right)\left|\Psi\left(x_{1}, t\right) \ldots \Psi\left(x_{n}, t\right)\right|^{2} d x_{1} \ldots d x_{n} \\
=-2 x_{0} a^{-2} \int_{-\infty}^{\infty} x_{1}\left|\Psi\left(x_{1}, t\right)\right|^{2} d x_{1} \ldots \int_{-\infty}^{\infty}\left|\Psi\left(x_{n}, t\right)\right|^{2} d x_{n}
\end{gathered}
$$




$$
\begin{gathered}
\ldots-2 x_{o} a^{-2} \int_{-\infty}^{\infty} x_{n}\left|\Psi\left(x_{1}, t\right)\right|^{2} d x_{1} \ldots \int_{-\infty}^{\infty}\left|\Psi\left(x_{n}, t\right)\right|^{2} d x_{n} \\
=n\left(-2 x_{o} a^{-2} \bar{x}\right)=-2 x_{o} a^{-2} n \bar{x} .
\end{gathered}
$$

O ultimo termo do lado direito da Eq. 11 pode ser reescrito, da seguinte forma

$$
a^{-2} \int_{-\infty}^{\infty}\left(\sum x_{k} x_{j}\right)\left|\Psi\left(x_{1}, t\right) \ldots \Psi\left(x_{n}, t\right)\right|^{2} d x_{1} \ldots d x_{n}=a^{-2}\left[n \overline{x^{2}}+n(n-1)(\bar{x})^{2}\right]
$$

pois teremos $a^{-2} \overline{x^{2}}$ quando $k=j$ oque acontece $n$ vezes, e teremos $a^{-2}(\bar{x})^{2}$ quando $k \neq j$, oque acontece $n(n-1)$ vezes.

Assim a Eq. (11) resulta em

$$
\begin{gathered}
a^{-2} x_{o}^{2}+a^{-2}\left[n \overline{x^{2}}+n(n-1)(\bar{x})^{2}\right]-2 x_{o} a^{-2} n \bar{x} \\
=a^{-2}\left[n \overline{x^{2}}+n^{2} \bar{x}^{2}-n \bar{x}^{2}+x_{o}^{2}-2 x_{o} n \bar{x}\right]=a^{-2}\left(x_{o}-n \bar{x}\right)^{2}+n\left(\overline{x^{2}}-\bar{x}^{2}\right)
\end{gathered}
$$

Neste ponto é conveniente introduzir uma nova notação. Denotemos

$$
(u, v)=\int_{-\infty}^{\infty} u(x) v(x)^{*} d x
$$

onde o asterisco denota o complexo conjugado. É fácil verificar que a Eq. (13) define um produto interno [4], induzindo uma norma

$$
\|u\|^{2} \equiv(u, u)
$$

Por exemplo, podemos reescrever alguns termos da Eq.(12), esta notação de produto interno, $\overline{x^{2}}=(x \Psi, x \Psi)=\|x \Psi\|^{2}$ e $\quad \bar{x}=(x \Psi, \Psi)$. 
Continuando nossa demonstração do Teorema 2.2 , teremos que

$$
a^{-2}\left(x_{o}-n \bar{x}\right)^{2}+n\left(\overline{x^{2}}-\bar{x}^{2}\right)=a^{-2}\left(x_{o}-n(x \Psi, \Psi)\right)^{2}+n\left(\|x \Psi\|^{2}-(x \Psi, \Psi)^{2}\right)
$$

Podemos ajustar esta equação para analisar a probabilidade de $\left|n \bar{x}-\sum x_{k}\right|>n \varepsilon$. Então faremos a seguinte troca na Eq. (12) $x_{o} \rightarrow n \bar{x} \mathrm{e} a \rightarrow n \varepsilon$. Deste modo, teremos que o lado direito da equação acima será dado por

$$
\varepsilon^{-2}(\bar{x}-(x \Psi, \Psi))^{2}+\frac{\varepsilon^{-2}}{n}\left(\|x \Psi\|^{2}-(x \Psi, \Psi)^{2}\right)
$$

Que tende a zero quando $n \rightarrow \infty$. O que implica que $\bar{x}-(x \Psi, \Psi) \rightarrow 0$, ou seja

$$
\bar{x}=(x \Psi, \Psi)
$$

O resultado do Teorema 2.2 pode ser estendido para tratar o valor esperado de funções da posição. Nestas condições enunciamos o teorema abaixo, sua demonstração é análoga a do Teorema 2.2.

Teorema 2.3 - Se $f: \mathbb{R} \rightarrow \mathbb{R}$ é uma função contínua e satisfaz $\int_{-\infty}^{\infty}|f(x)||\Psi(x, t)|^{2} d x<\infty$, então o valor médio de $f(x)$ é dado por

$$
\overline{f(x)}=\int_{-\infty}^{\infty} f(x)|\Psi(x, t)|^{2} d x
$$

\subsection{Momento Linear}

Novamente considerando o caso da Luz, Compton mostrou que o momento linear associado a um fóton é 


$$
p=\hbar k
$$

onde $k=\frac{2 \pi}{\lambda}$ é o número de onda, $p$ é o momento do fóton e $\lambda$ é o comprimento de onda da luz. O conjunto das Eq.(1) e (15) são as famosas relações de De Broglie, que as propôs para descrever o comportamento ondulatório da matéria (não só de radiações!). Note que sendo $h$ é uma constante de valor muito pequeno, o comprimento de onda de De Broglie de objetos do nosso cotidiano se torna desprezível, sendo significativo apenas para objetos de dimensões atômicas que possuem massas muito menores do que as que estamos habituados em nosso cotidiano. Para lidarmos com a interpretação quântica do momento linear, estabeleceremos o

Postulado 2- A probabilidade do momento $p$ de uma partícula estar contido em um intervalo $I$ é dado por

$$
\frac{1}{\mathrm{~h}} \int_{I}\left|\widehat{\Psi}\left(\frac{p}{\mathrm{~h}}, t\right)\right|^{2} d p
$$

onde h é a constante de Planck e $\widehat{\Psi}$ é a transformada de Fourier da função de onda $\Psi$ da partícula.

A transforma de Fourier, e.g. [6], é dada por

$$
\widehat{\Psi}(k, t)=\frac{1}{\sqrt{2 \pi}} \int_{-\infty}^{\infty} e^{-i k x} \Psi(x, t) d x
$$

Analogamente ao Teorema 2.2 esperamos que $\bar{p}=\frac{1}{\mathrm{~h}} \int_{-\infty}^{\infty} p\left|\widehat{\Psi}\left(\frac{p}{\mathrm{~h}}, t\right)\right|^{2} d p$.

Das relações de De Broglie $p=\hbar k$ e $d p=\hbar d k$, logo substituindo na integral acima teremos, 


$$
\bar{p}=\hbar \int_{-\infty}^{\infty} k|\widehat{\Psi}(k, t)|^{2} d k
$$

O Teorema 2.3 também tem sua versão correspondente para o momento.

Teorema 2.4 - Se $g(p)$ é contínua e satisfaz $\int_{-\infty}^{\infty}|g(\hbar k)||\widehat{\Psi}(k, t)|^{2} d k<\infty$, então

$$
\overline{g(p)}=\int_{-\infty}^{\infty} g(\hbar k)|\widehat{\Psi}(k, t)|^{2} d k
$$

Como se pode perceber será conveniente utilizar transformada de Fourier, as propriedades da mesma estão presentes em [6], uma delas segue abaixo:

Propriedade 1)

$$
\left[\frac{\partial \Psi(x, t)}{\partial x}\right]^{\sim}=i k \widehat{\Psi}(k, t)
$$

Demonstração da Propriedade 1:

$$
\left[\frac{\partial \Psi(x, t)}{\partial x}\right]^{-}=\frac{1}{\sqrt{2 \pi}} \int_{-\infty}^{\infty} e^{-i k x} \frac{\partial \Psi(x, t)}{\partial x} d x
$$

Usando integração por partes, tomando $u=e^{-i k x}$ e $d v=\frac{\partial \Psi(x, t)}{\partial x} d x$, teremos,

$$
\sqrt{2 \pi}\left[\frac{\partial \Psi(x, t)}{\partial x}\right] \sim=\left[e^{-i k x} \Psi(x, t)\right]_{-\infty}^{\infty}-\int_{-\infty}^{\infty} \Psi(x, t)\left(-i k e^{-i k x}\right) d x .
$$

Note que como $\Psi(x, t)$ é quadraticamente integrável (Eq. 3), precisaremos que $\lim _{x \rightarrow \pm \infty} \Psi(x, t)=0$, como $e^{-i k x}$ é limitada, conclui-se que

$$
\sqrt{2 \pi}\left[\frac{\partial \Psi(x, t)}{\partial x}\right]^{-}=-\int_{-\infty}^{\infty} \Psi(x, t)\left(-i k e^{-i k x}\right) d x=i k \int_{-\infty}^{\infty} e^{-i k x} \Psi(x, t) d x .
$$


Logo,

$$
\left[\frac{\partial \Psi(x, t)}{\partial x}\right]^{\sim}=i k \widehat{\Psi}(k, t)
$$

Podemos reescrever a equação 17 para o momento médio da seguinte forma:

$$
\bar{p}=\hbar \int_{-\infty}^{\infty} k \widehat{\Psi}(k, t) \widehat{\Psi}(k, t)^{*} d k
$$

Aplicando a propriedade 1, podemos reescrever a Eq. 19, obtendo

$$
\bar{p}=-i \hbar \int_{-\infty}^{\infty}\left[\frac{\partial \Psi(x, t)}{\partial x}\right] \widetilde{\Psi}(k, t)^{*} d k
$$

E usando a Identidade de Parseval, e.g. [6], podemos substituir a integral das transformadas pela integral das transformadas inversas:

$$
\bar{p}=-i \hbar \int_{-\infty}^{\infty}\left[\frac{\partial \Psi(x, t)}{\partial x}\right] \Psi(x, t)^{*} d x=\int_{-\infty}^{\infty} \hat{P}[\Psi(x, t)] \Psi(x, t)^{*} d x
$$

onde $\hat{P}=-i \hbar \frac{\partial}{\partial x}$. O operador $\hat{P}$ é chamado operador momento. Vale apena notar que a aplicação repetida da propriedade 1, confirma o teorema 2.4, implicando que $\left[\frac{\partial^{n} \Psi(x, t)}{\partial x^{n}}\right]^{\sim}=(i k)^{n} \widehat{\Psi}(k, t)$. Consequentemente, temos que:

$$
\overline{p^{n}}=\left(\widehat{P}^{n} \Psi, \Psi\right)
$$

onde utilizamos a notação de produto interno definida na Eq. (13). 


\subsection{Energia}

Na Física Clássica a energia cinética $T$ de uma partícula é dada por $T=\frac{p^{2}}{2 m}$. Da Eq.(20) sabemos que podemos obter o valor médio da energia cinética da seguinte forma,

$$
\bar{T}=\frac{1}{2 m}\left(\hat{P}^{2} \Psi, \Psi\right)
$$

Uma vez que $\overline{p^{2}}=\left(\hat{P}^{2} \Psi, \Psi\right)$. A energia total do sistema é dada por $E=\frac{p^{2}}{2 m}+V(x)$, onde $V: \mathbb{R} \rightarrow \mathbb{R}$ é a função da energia potencial associada. Se $V$ satisfaz o Teorema 2.3, então o valor médio da energia potencial será dado por:

$$
\bar{V}=\int_{-\infty}^{\infty} V(x)|\Psi(x, t)|^{2} d x
$$

Teorema 2.5- O valor médio de uma soma é igual à soma dos valores médios.

Demonstração - Seja $u$ uma quantidade mensurável que pode assumir os valores $u_{1}, \ldots, u_{m}$ com respectivas probabilidades $P_{1}, \ldots, P_{m}$, e seja $v$ também uma quantia mensurável que assume os valores $v_{1}, \ldots, v_{n}$ com as respectivas probabilidades $Q_{1}, \ldots, Q_{n}$. Seja $R_{i j}$ a probabilidade de que simultaneamente ocorra $u=u_{i}$ e de que $v=v_{j}$. Se as medidas são independentes então $R_{i j}=P_{i} Q_{j}$. Dessa forma $P_{i}=\sum_{j} R_{i j}$ e $Q_{j}=\sum_{i} R_{i j}$. Logo o valor médio de $u+v$ é dado por

$$
\overline{u+v}=\sum_{i, j}\left(u_{i}+v_{j}\right) R_{i j}=\sum_{i, j} u_{i} R_{i j}+\sum_{i, j} v_{j} R_{i j}=\sum_{i} u_{i} P_{i} \sum_{j} Q_{j}+\sum_{j} v_{j} Q_{j} \sum_{i} P_{i}
$$

Utilizando que $\sum_{j} Q_{j}=\sum_{i} P_{i}=1$, temos 


$$
\overline{u+v}=\sum_{i} u_{i} P_{i}+\sum_{j} v_{j} Q_{j}
$$

E pela Eq. 23, podemos reescrever a equação $\overline{u+v}=\bar{u}+\bar{v}$, o que conclui a demonstração.

Do Teorema 2.5, podemos escrever a energia total média da seguinte forma:

$$
\bar{E}=\overline{\frac{p^{2}}{2 m}+V(x)}=\frac{1}{2 m} \overline{p^{2}}+\overline{V(x)}
$$

Substituindo as médias pelas equações 21 e 22 :

$$
\begin{aligned}
& \bar{E}=-\frac{\hbar^{2}}{2 m} \int_{-\infty}^{\infty}\left[\frac{\partial^{2} \Psi(x, t)}{\partial x^{2}}\right] \cdot \Psi(x, t)^{*} d x+\int_{-\infty}^{\infty} V(x) \Psi(x, t) \Psi(x, t)^{*} d x \\
& =\int_{-\infty}^{\infty}\left\{-\frac{\hbar^{2}}{2 m} \frac{\partial^{2} \Psi(x, t)}{\partial x^{2}}+V(x) \Psi(x, t)\right\} \Psi(x, t)^{*} d x \\
& =\int_{-\infty}^{\infty}\left\{-\frac{\hbar^{2}}{2 m} \frac{\partial^{2}}{\partial x^{2}}+V(x)\right\} \Psi(x, t) \Psi(x, t)^{*} d x \\
& =\int_{-\infty}^{\infty} \widehat{H} \Psi(x, t) \Psi(x, t)^{*} d x=(\widehat{H} \Psi, \Psi),
\end{aligned}
$$

onde $\widehat{H}$ é o operador energia ou Hamiltoniano, dado por:

$$
\widehat{H}=-\frac{\hbar^{2}}{2 m} \frac{\partial^{2}}{\partial x^{2}}+V(x)=\frac{\hat{P}^{2}}{2 m}+V(x)
$$

\subsection{Observáveis}

Qualquer quantidade que pode ser medida é denominada um observável. Nós já discutimos três destas quantidades, são elas, posição, momento e energia. Vimos 
que, de maneira geral, se temos uma quantidade variável $a$, então o seu valor médio $\bar{a}$ é dado por $\bar{a}=(\hat{A} \Psi, \Psi)$, onde $\hat{A}$ é um operador correspondente ao observável. Isto fica mais claro olhando a seguinte tabela

Tabela 1. Observáveis e respectivos Operadores

\begin{tabular}{|l|l|}
\hline$a$ & $\hat{A}$ \\
\hline$x$ & $\hat{x}=x$ \\
\hline$p$ & $\hat{P}=-i \hbar \frac{\partial}{\partial x}$ \\
\hline$E$ & $\hat{H}=-\frac{\hbar^{2}}{2 m} \frac{\partial^{2}}{\partial x^{2}}+V$ \\
\hline
\end{tabular}

Podemos verificar na tabela os observáveis e o operador correspondente à cada um, os quais serão usados quando calcularmos o valor esperado de cada observável. Em cada um dos casos mencionados, podemos notar que o observável assume apenas valores reais. Logo, o valor médio de um observável também deve assumir valores reais. Isto implica que $(\hat{A} \Psi, \Psi)$ deve assumir valores reais para qualquer $\Psi$. Portanto devemos nos perguntar: "quais operadores $\hat{A}$ satisfazem a propriedade de que $(A \Psi, \Psi)$ é real para qualquer função $\Psi$ ?". Passaremos agora a analisar esta questão.

Um operador $\hat{A}$ é chamado Hermiteano se

$$
(\hat{A} \Psi, \varphi)=(\Psi, \hat{A} \varphi)
$$

para todo $\Psi$ e $\varphi$. A resposta para a pergunta feita anteriormente é dada pelo: 
Lema 2.7- Um operador $\hat{A}$ é Hermiteano se e somente se $(\hat{A} \Psi, \Psi) \in \mathbb{R}$ para todo $\Psi$. Antes de demonstrarmos o lema 2.7, estudemos melhor alguns conceitos e definições, por exemplo, as propriedades do produto interno, que se encontram em [5], uma dessas propriedades segue abaixo:

Propriedade 2 - $\|\Psi\|^{2} \equiv(\Psi, \Psi)>0$, exceto para $\Psi=0$.

Observação - A propriedade 2 é válida em quase todo o ponto, funções que sejam nulas exceto em um número finito de pontos obedecem $(\Psi, \Psi)=0$. Mas como será discutido mais adiante, nós consideraremos iguais duas funções que são iguais em quase todos os pontos.

Uma questão importante é, quais as funções aceitáveis para o papel de função de onda ou função de estado? Devemos nos lembrar de que uma candidata à função $\Psi$ deve satisfazer a equação 3, o que implica que a função deve ser quadraticamente integrável em $(-\infty, \infty)$. O espaço das funções quadraticamente integráveis é denominado $L^{2} \equiv L^{2}(-\infty, \infty)$.

Seja $\left\{\Psi_{k}\right\}$ uma sequência de funções em $L^{2}$ tais que $\left\|\Psi_{j}-\Psi_{k}\right\| \rightarrow 0$ quando $j, k \rightarrow \infty$. Então existe $\Psi \in L^{2}$, tal que $\left\|\Psi_{k}-\Psi\right\| \rightarrow 0$, quando $k \rightarrow \infty$. Espaços como $\circ L^{2}$, que exibem esta propriedade são chamados de completos. Em palavras, a completeza significa que toda sequencia de Cauchy em $L^{2}$ converge para um elemento em $L^{2}$

Quando $\left\|\Psi_{k}-\Psi\right\| \rightarrow 0$, temos a chamada convergência forte ou convergência na norma, e indicaremos este fato denotando simplesmente $\Psi_{k} \rightarrow \Psi$. Dizemos que $\Psi_{k}$ converge fracamente para $\Psi_{1}$ e denotamos $\Psi_{k} \rightarrow \Psi$, se 
$\left(\Psi_{k}, \varphi\right) \rightarrow(\Psi, \varphi)$, quando $k \rightarrow \infty$ para todo $\varphi \in L^{2}$. Note que convergência forte implica em convergência fraca. Isto é uma consequência da Desigualdade de Schwarz [5] a qual noz diz que $|(f, g)| \leq\|f\|\|g\|$, e que possui como consequência imediata a chamada Desigualdade Triangular [5] que nos diz que $|f+g| \leq\|f\|+\|g\|$.

A relação entre a convergência forte e a convergência fraca deve agora se tornar mais clara. Se $\left\|\Psi_{k}-\Psi\right\| \rightarrow 0$, quando $k \rightarrow \infty$, então para todo $\varphi \neq 0$ temos que, $\left\|\Psi_{k}-\Psi\right\|\|\varphi\| \rightarrow 0$. Então da Desigualdade de Schwarz, segue que $\left\|\Psi_{k}-\Psi\right\|\|\varphi\| \geq\left\|\left(\Psi_{k}-\Psi, \varphi\right)\right\| \rightarrow 0$. Isto implica que $\left(\Psi_{k}-\Psi, \varphi\right) \rightarrow 0$ e portanto $\left(\Psi_{k}, \varphi\right) \rightarrow(\Psi, \varphi)$.

Um subespaço $S$ de $L^{2}$ é chamado fechado se para toda sequência de funções em $S$ a qual converge em $L^{2}$, o limite é também de $S$. E um subespaço $S$ de $L^{2}$ pode ser chamado de denso se para todo $\varepsilon>0$ e todo $\Psi \in L^{2}$, temos $\varphi \in S$ tal que $\|\Psi-\varphi\|<\varepsilon$.

Esta ideia de subespaço denso pode ser melhor compreendida no seguinte lema:

Lema 2.10: Se $S$ é denso em $L^{2}$ e $(\Psi, \varphi)=0$, para todo $\varphi \in S$, então $\Psi=0$.

Demonstração- Para $\quad \varphi \in S$, temos por hipótese $\|\Psi-\varphi\|^{2}=(\Psi-\varphi, \Psi-\varphi) \geq\|\Psi\|^{2}+\|\varphi\|^{2} \geq\|\Psi\|^{2}$. Como $S$ é denso, então $\|\Psi-\varphi\|$ pode ser feito tão pequeno quanto se queira. Logo $\|\Psi\|$ também precisa se tornar arbitrariamente pequeno, portanto $\Psi=0$.

Demonstração do Lema 2.7: Pelas propriedades do produto interno temos que $(\Psi, \hat{A} \Psi)=(\hat{A} \Psi, \Psi)^{*}$, se $\hat{A}$ é hermitiano $(\hat{A} \Psi, \Psi)=(\Psi, \hat{A} \Psi)$, de onde segue que 
$(\hat{A} \Psi, \Psi)=(\hat{A} \Psi, \Psi)^{*}$ e portanto $(\hat{A} \Psi, \Psi) \in \mathbb{R}$. Por outro lado, tomando $\psi+i \varphi$, usamos novamente as propriedades de produto interno para obter $(\hat{A}(\psi+i \varphi), \psi+i \varphi)=(\hat{A} \psi, \psi)+(\hat{A} \varphi, \varphi)-i(\hat{A} \psi, \varphi)+i(\hat{A} \varphi, \psi)$.

Se $(\hat{A} \Psi, \Psi) \in \mathbb{R}$ para todo $\Psi$, então $(\hat{A} \psi, \varphi)-(\hat{A} \varphi, \psi)=i r$, logo para todo par $\psi$ e $\varphi$,

$$
\operatorname{Re}(\hat{A} \psi, \varphi)=\operatorname{Re}(\hat{A} \varphi, \psi)=\operatorname{Re}(\psi, \hat{A} \varphi)^{*}=\operatorname{Re}(\psi, \hat{A} \varphi),
$$

onde usamos que $\operatorname{Re} z=\operatorname{Re} z^{*}$. Por outro lado, a Eq.(26) implica que

$$
\begin{aligned}
& \operatorname{Im}(\hat{A} \psi, \varphi)=\operatorname{Im}[-i(\hat{A} i \psi, \varphi)]=-\operatorname{Re}(\hat{A} i \psi, \varphi)=-\operatorname{Re}(i \varphi, \hat{A} \psi) \\
& =\operatorname{Im}(\hat{A} \varphi, \psi),
\end{aligned}
$$

onde usamos que $I m-i z=-\operatorname{Re} z$ e Re $i z=-\operatorname{Im} z$. O resultado segue combinando Eq.(26) e a Eq.(27).

\subsection{Operadores}

Temos visto que para cada observável $a$, existe um operador $\hat{A}$, tal que $\bar{a}=(\hat{A} \Psi, \Psi)$. Por exemplo, no caso do observável momento linear temos que o operador correspondente é $\widehat{P}=-i \hbar \frac{\partial}{\partial x^{\prime}}$ o qual nos auxilia a encontrar $\bar{p}$ da seguinte forma

$$
\bar{p}=(\hat{P} \Psi, \Psi)=\int_{-\infty}^{\infty}-i \hbar \frac{\partial \Psi}{\partial x} \Psi^{*} d x
$$


O integrando nos diz que $\Psi$ deve ser diferenciável com relação a $x$, e o momento médio tem valor finito somente se a integral existe e converge. Então o domínio do operador $\widehat{P}, D(\widehat{P})$, não consiste necessariamente de todo $\Psi \in L^{2}$. Ou seja, existem $\Psi \in L^{2}$ que não são diferenciáveis. $O$ fato de $\Psi$ não ser diferenciável pode ser resolvido usando o fato de que $D(\widehat{P})$ é denso em $L^{2}$, ou seja, podemos usar uma sequência do $D(\hat{P})$ para aproximarmos o quanto quisermos de $\Psi$. Podemos usar, por exemplo, séries de Fourier para aproximar estas funções. Podemos entender melhor estas ideias a partir do

Postulado 3 - Para todo observável a corresponde um operador Â com domínio denso tal que:

$$
\bar{a}=(\hat{A} \Psi, \Psi)
$$

Válido para todo $\Psi \in D(\hat{A})$. Se $\widehat{B}$ é um operador hermiteano tal que $D(\hat{A}) \subset$

$$
\begin{gathered}
D(\widehat{B}) \mathrm{e} \\
\bar{a}=(\widehat{B} \Psi, \Psi)
\end{gathered}
$$

Sempre que $\Psi \in D(\widehat{A})$, então $\widehat{B}=\widehat{A}$. Em consequência do Lema 2.7 , nós temos o

Teorema 2.6 - Se o observável a assume valores reais, então o operador correspondente é hermiteano.

Podemos verificar este teorema para os operadores $\hat{x}, \widehat{P}$ e $\widehat{H}$ e mostrar que eles são de fato hermiteanos, é o que faremos agora.

Posição: como $x \in \mathbb{R}$, temos que $x=x^{*}$, logo, 


$$
(x \Psi, \Psi)=\int_{-\infty}^{\infty} x \Psi \Psi^{*} d x=\int_{-\infty}^{\infty} \Psi\left(x^{*} \Psi^{*}\right) d x=(\Psi, x \Psi)
$$

Momento linear:

$$
(\tilde{P} \Psi, \Psi)=\int_{-\infty}^{\infty}-i \hbar \frac{\partial \Psi}{\partial x} \Psi^{*} d x
$$

Integrando por partes teremos,

$$
\int_{-\infty}^{\infty}-i \hbar \frac{\partial \Psi}{\partial x} \Psi^{*} d x=\left[-i \hbar \Psi \Psi^{*}\right]_{-\infty}^{\infty}+\int_{-\infty}^{\infty} \Psi\left(-i \hbar \frac{\partial \Psi}{\partial x}\right)^{*} d x
$$

o primeiro termo da direita deve anular-se, assim

$$
(\hat{P} \Psi, \Psi)=\int_{-\infty}^{\infty}-i \hbar \frac{\partial \Psi}{\partial x} \Psi^{*} d x=\int_{-\infty}^{\infty} \Psi\left(-i \hbar \frac{\partial \Psi}{\partial x}\right)^{*} d x=(\Psi, \hat{P} \Psi)
$$

Energia: primeiro, sabendo que $\widehat{P}$ é hermiteano então $\left(\hat{P}^{2} \Psi, \Psi\right)=(\hat{P} \Psi, \hat{P} \Psi)=\left(\Psi, \hat{P}^{2} \Psi\right), \log \circ$

$$
\left(\widehat{P}^{2} \Psi, \Psi\right)=\left(\Psi, \widehat{P}^{2} \Psi\right)
$$

potencial $V(x)$ pode ser escrito como uma expansão em série de taylor, da seguinte forma $V(x)=\sum a_{n} x^{n}$, como sabemos, $x \in \mathbb{R}$, logo $a_{j} x^{j}=\left(a_{j} x^{j}\right)^{*}$, assim, $(V \Psi, \Psi)=\left(\sum a_{n} x^{n} \Psi, \Psi\right)=\left(\Psi, \sum a_{n} x^{n} \Psi\right)=(\Psi, V \Psi), \log \circ$

$$
(V \Psi, \Psi)=(\Psi, V \Psi)
$$


Agora sim, provando que o operador Hamiltoniano é hermiteano

$$
(\widehat{H} \Psi, \Psi)=\left(\left[\frac{\widehat{P}^{2}}{2 m}+V\right] \Psi, \Psi\right)=\left(\frac{\widehat{P}^{2}}{2 m} \Psi, \Psi\right)+(V \Psi, \Psi)
$$

Usando as equações 28 e 29 , teremos que

$$
\left(\frac{\widehat{P}^{2}}{2 m} \Psi, \Psi\right)+(V \Psi, \Psi)=\left(\Psi, \frac{\hat{P}^{2}}{2 m} \Psi\right)+(\Psi, V \Psi)=\left(\Psi,\left[\frac{\widehat{P}^{2}}{2 m}+V\right] \Psi\right)=(\Psi, \widehat{H} \Psi)
$$

O que conclui a demonstração.

Suponha agora um $\Psi \notin D(\hat{A})$, porém existe uma sequência $\left\{\Psi_{n}\right\} \subset D(\hat{A})$, tal que $\Psi_{n} \rightarrow \Psi$ e $\hat{A} \Psi_{n} \rightarrow f$. Então poderemos definir $\hat{A} \Psi$ como sendo $f$. É claro que para isto fazer sentido, $f$ não pode depender da sequência $\left\{\Psi_{n}\right\}$. Pois se $\left\{\Psi_{n}^{\prime}\right\} \subset D(\hat{A})$ é tal que $\Psi_{n}^{\prime} \rightarrow \Psi$ e $\hat{A} \Psi_{n}^{\prime} \rightarrow g$, devemos ter $g=f$. Ou dito de outra maneira, se $\left\{\varphi_{n}\right\} \subset D(\hat{A})$ satisfaz $\varphi_{n} \rightarrow 0$ e $\hat{A} \varphi_{n} \rightarrow w$, devemos ter $w=0$ para que este método funcione. Um operador que possui estas propriedades é chamado fechavel ou pré-fechado. O operador estendido é chamado fecho ou fechamento, e as vezes denotado por $\bar{A}$. Ela tem a seguinte propriedade.

Lema 2.11 - Se $\left\{\Psi_{n}\right\} \subset D(\hat{A}), \Psi_{n} \rightarrow \Psi, \hat{A} \Psi_{n} \rightarrow f$, então $\Psi \in D(\hat{A})$ e $\bar{A} \Psi=f$.

Demonstração: Para todo $\Psi_{n} \in D(\bar{A})$ existe uma sequência $\Psi_{n k} \in D(\hat{A})$, tal que $\Psi_{n k} \rightarrow \Psi_{n}$, e $\hat{A} \Psi_{n k} \rightarrow \hat{A} \Psi_{n}$. Como $\Psi_{n} \rightarrow \Psi$ e $\hat{A} \Psi_{n} \rightarrow f$, então a sequência $\Psi_{k k} \rightarrow \Psi$ e $\hat{A} \Psi_{k k} \rightarrow f$. Logo $\Psi$ pode ser atingido por uma sequência de elementos de $D(\hat{A})$ e portanto $\Psi \in D(\bar{A})$ e $\bar{A} \Psi=f$. 
Portanto o operador $\hat{A}$ não pode ser mais extendido pelo método descrito acima. Um operador que possui as propriedades descritas no Lema 2.11 é chamado fechado. Nem todos os operadores são fecháveis, mas temos

Lema 2.12 - Um operador Hermiteano com dominio denso é fechável.

Demonstração: Se $\varphi_{n} \in D(\hat{A}), \quad \varphi_{n} \rightarrow 0, \quad \hat{A} \varphi_{n} \rightarrow w, \quad$ então para qualquer $h \in D(\hat{A})$,teremos que $\left(\hat{A} \varphi_{n}, h\right)=\left(\varphi_{n}, \hat{A} h\right)$, tomando o limite desta equação notamos que $(w, h)=0$ para todo $h \in D(\hat{A})$. Podemos agora aplicar o Lema 2.10 e concluir que $w=0$. Esta discussão nos leva ao resultado mais importante desta seção enunciado no

Teorema 2.7 - O operador $\hat{A}$ correspondente ao observável real $a$ é fechado.

Demonstração: $\hat{A}$ é densamente definido de acordo com o Postulado 3 e Hermiteano pelo teorema 2.6. Assim ele é fechavel pelo lema 2.11. $\bar{A}$ é Hermiteano, pois se $\left\{\Psi_{n}\right\},\left\{\varphi_{n}\right\} C D(\hat{A}), \quad \Psi_{n} \rightarrow \Psi, \varphi_{n} \rightarrow \varphi, \quad \hat{A} \Psi_{n} \rightarrow \bar{A} \Psi, \quad \hat{A} \varphi_{n} \rightarrow \bar{A} \varphi, \quad$ então $(\bar{A} \Psi, \varphi)=\lim \left(\hat{A} \Psi_{n}, \varphi_{n}\right)=\lim \left(\Psi_{n}, \hat{A} \varphi_{n}\right)=(\Psi, \bar{A} \varphi)$, concluímos então que pelo postulado $3 \hat{A}=\bar{A}$, por isso é fechado pelo Lema 2.11.

\section{Conclusões}

Neste texto foram discutidas definições básicas da mecânica quântica, introduzindo as ideias de operadores, domínio denso e fechamento de operadores aplicadas a mesma. Foram enunciados teoremas e suas respectivas demonstrações, buscando manter a clareza e o rigor matemático. 


\section{Agradecimentos}

O autor Lucas Elias Viera agradece ao apoio financeiro do CNPq através da bolsa iniciação científica PIBIC/UFG. 


\section{Operator Methods in Quantum Mechanics}

Abstract: This paper will introduce and discuss the method of Operators in Quantum Mechanics. This presentation will be mathematically rigorous, but also physically motivate the definitions and propositions established.

\section{Keywords: Physics; Quantum Mechanics; Operators.}

\section{Referências bibliográficas}

Schechter, Martin. Operator methods in quantum mechanics. Courier Dover Publications, 2011.

Resnick, Robert, and Robert Eisberg. "Física Quântica-Átomos, Moléculas, Sólidos, Núcleos e Partículas." Editora Campus (1979).

Feynman, Richard Phillips, Robert B. Leighton, and Matthew Linzee Sands. The Feynman Lectures on Physics: Mainly Mechanism, Radiation and Heat. Addison Wesley, 1963.

Feller, William. "Probability theory and its applications." New York (1950)

Lima, Elon Lages. Álgebra linear. IMPA, 2006.

Butkov ,Eugene."Fısica matemática." Rio de Janeiro: Livros Técnicos e Cientıficos (1988). 